\title{
Our World is Well Ordered in Measurement and Number - Or Why Natural Constants Are as They Are
}

\author{
Walter Alfred van Laack \\ University of Applied Sciences, Aachen, Campus Juelich, Germany
}

\begin{abstract}
All the important natural constants can be logically explained with and derived from the first four ordinal numbers, 1, 2, 3 and 4, its addition to ten and finally the standard values for obviously maximal feasibility $\Omega$ and the optimum in our world, the Golden Section (GS), i.e. the number sequences 273 and 618 . They both are the first three numbers of irrational results by an arithmetical transformation of simple geometrical relationships by creating multiplicity out of singularity. Both of them show that the infinite is inherent in finiteness and explain in a simple way the smallest deviations and fluctuations between the physical AS-IS state and the obvious spiritual ideal behind: Wherever we look in this world, and especially in important key-positions, we regularly find these sequences. All of the above mentioned numbers so seem to be key players in our world, what can be demonstrated by the derivation of natural constants.
\end{abstract}

Keywords: Natural constants, universe in order

\section{Introduction}

A little intellectual experiment that only needs some simple geometry will be able to help us achieving a completely widened view of the world. Imagining being the "Creator", taking a sheet of paper (=two dimensions) and starting drawing, consequently according to two simple guidelines:

1) The starting point of all this is the finite singularity of a circle, It now should grow, i.e. increase in size, and also propagate itself, i.e. increase in number, in free adaption of the biblical motto: "Be fruitful and multiply".
2) Every new introduction should be unequivocally retraceable to already existing information whereby every new invention must simultaneously strive for perfection.

In the first step the first circle (C1) expands and creates the second $(\mathrm{C} 2)$ which is polar-symmetrical to the finite singularity. That allows the creation of a rectangular triangle (RT) that cuts the (new) second circle on its circular arch. Thus a new point of information is given which now facilitates the creation of the first larger circle (LC1). The larger circle cuts the rectangular triangle on a cathetus and creates the "Golden Section" (GS), what leads to the number sequence $6-1-8(1,618 \ldots$ or $0,618 \ldots)$.

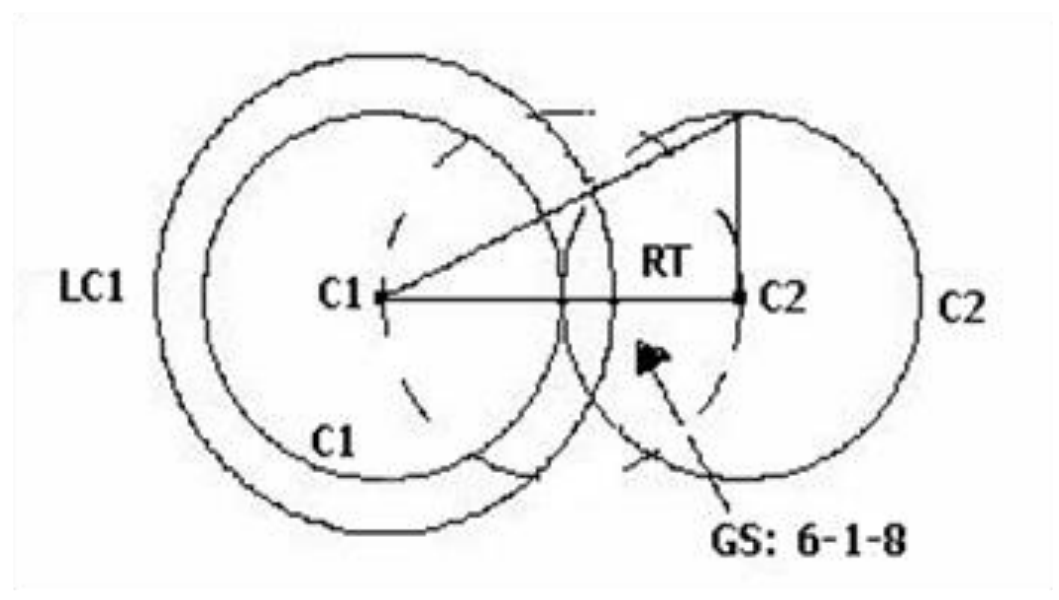

After in total four steps, reflecting the first four ordinal numbers ${ }^{1}$, we come to a square by connecting the four central points of the meanwhile four created circles. This square $(S)$ is the first new perfection in multiplicity. It surrounds the basic circle. By dividing the area of the circle $(\mathrm{C})$ we will get an infinite, i.e. irrational number with the sequence $2-7$ 3 , what I call $\Omega$. 
The addition of the first four ordinal numbers that are involved up to this point (four circles create the square) leads to the number 10 . It seems no mere coincidence that in this world we prefer the decimal system for calculations. And although the above achieved irrational numbers for $\Omega$ and $G S$ are the results of using the decimal system, they base upon geometrical relations. Other calculation systems may indeed lead to different numbers, but then equally for all other applications and models: The ratios would not be different.

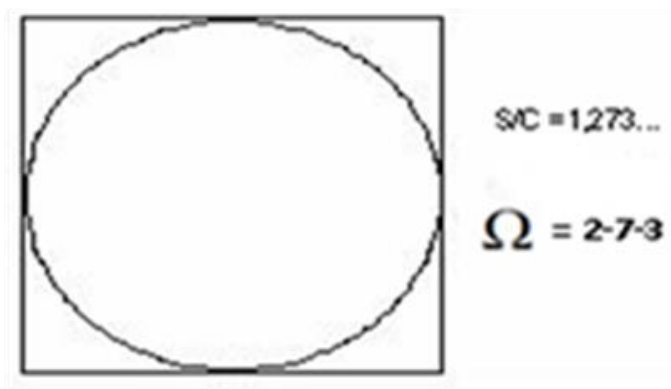

By means of a simple geometrical growth and propagation process and by way of two "transcendental" geometrical ratios $(1.618 \ldots$ and $1.273 \ldots)$ we arrive at the very important number sequences 2-7-3 and 6-1-8.

Both are irrational numbers and thus perhaps an expression of the imperfect implementation of the spirit in the physical world that often is measured as very slight fluctuations (e.g. the universe's mostly isotropic background radiation of $2,73 \ldots \mathrm{K}$ ). The number sequence 273 seems to be the constant for the maximum expansion within the bounds of feasibility - the reason why I call it $\Omega$ - whilst the number $618(G S)$ seems to stand for optimal realizations, i.e. for perfection in nature.

If the most important so-called natural constants are now considered from this point of view, amazing things become apparent:

All natural constants are rigid physical quantities which can be measured experimentally and which describe those special key parameters found in science for the existence of all matter in the physical universe as we know it. One of the declared aims of dedicated scientists is, of course, to connect these numbers in a rational way, i.e. to develop a common universal theory.

But, they have not yet succeeded in doing so. At first glance my ideas in this direction also seem rather farfetched. Nonetheless, I believe this is a legitimate and also plausible attempt to lend support to my hypothesis of combination which is consistently based on simple mathematical logical considerations.

Each of these natural constants fluctuates only very slightly around the measured value - at the very most by a few percentage points. Were they not as stable as they obviously are - and that is undisputed among all scientists involved - then our universe could not exist, nor could atoms or solid matter, nor could galaxies or planetary systems and planets, and, of course, the existence of life would be impossible.

I will show that all the following very important natural constants can be achieved by logically using the first four ordinal numbers that were necessary to create something new in multiplicity, starting in singularity and the two most important results $\Omega$ and $G S$ that came up on the way due to geometrical relations.

1) A very important natural constant is the velocity of light $c$.

It is thanks to Albert Einstein that this is recognised as being the absolute limit for speed in vacuum. Its measured value is $2.99792458 \times 10^{8} \mathrm{~m} / \mathrm{s}$, i.e. nearly 300,000 kilometres per hour. In all probability the ordinal number 3 actually stands behind this measured value and is thus the actual decisive, orientating "spiritual" value: In fact the first circle, the starting finite singularity in my intellectual game of being the creator, is clearly defined by at least 3 coordinates of its arc, generally by 3 points of (spiritual) information before manifesting as (material) "drawing".

The speed of light is a constant which also attains the "ideal" on which it is obviously based as close as makes no difference, this being the decimal multiple of the ordinal number 3 . This ideal renders it a constant. The divergence of the actual measured value from the number 3 is a mere $0.069 \%$.

Light is not created by the interaction of two bodies. It is rather related to the expansion of space in the universe which is expressed by the product 3 $\mathrm{x} 10^{\mathrm{n}}$. In contrast the effects between interdependent spatial bodies are expressed by the reciprocal value of this product.

Instead of $3 \times 10^{\mathrm{n}}$ these are expressed by the reciprocal, i.e. the factor:

$1:\left(3 \times 10^{\mathrm{n}}\right)$ or $1 / 3 \times 10^{-\mathrm{n}}$.

2) Gravitation is such a force. It is always in force between at least two (three-dimensional) bodies.

It follows that we can form a product by multiplying the number 2 by the reciprocal value mentioned above under 1$)$, i.e.:

$2 \times\left(1 / 3 \times 10^{-n}\right)$ or $2 / 3 \times 10^{-n}$ which equals 6.6666.. $\times$ $10^{-\mathrm{n}}$.

After the velocity of light the most important constant in our universe is the gravitation constant. It has the value $\mathrm{G}=6.67259 \times 10^{-19}\left(\mathrm{Nm}^{2} / \mathrm{kg}^{2}\right)$ which shows a deviation of only $0.088 \%$ from its calculated value.

3) Planck's elementary quantum of action, also known as Planck's constant $(h)$ is similar. It expresses the constant measure of the smallest effect between two bodies in our universe.

Again the same factor $\left(1 / 3 \times 10^{-n}\right)$ must play a role since it is the reciprocal value of the constant of spatial expansion and the velocity of light and stands for all effects working inwards. Since it is a force between two bodies it must again be multiplied by 2 (i.e. $2 / 3 \times 10^{-n}$ ). In fact its measured value is $\mathrm{h}=$ 
$6.626075 \cdot 10^{-34}(\mathrm{~J} / \mathrm{s}){ }^{2}$ The deviation here is only $0.61 \%$.

4) The two most important nuclear particles, the "proton" and the "electron" may be considered as being polar to each other. The proton carries a positive and the electron a negative charge. The hydrogen atom, which is by far the most important and most common atom in the entire universe, contains only these two. And they are in fact two extreme opposites - not only with regard to their opposing charges but also especially with regard to the difference in their sizes. This is also expressed in the proportions of their masses to one another. This is known as the mass quotient. It is also a natural constant and amounts to $1,836.152701$.

Is it not astonishing that the factor $2 / 3 \times 10^{\mathrm{n}}$ (where $\mathrm{n}=1$ ) which I mentioned above, multiplied by the parameter for maximum expansion, the feasibility, i.e. 273 , gives very nearly the same result again?

It is: $2 / 3 \times 10^{1} \times 273=1,820.9$. Rounded up, the deviation is only $0.84 \%$.

5) The elementary charge is also an important natural constant.

It was certainly meant to be an optimal value. To demonstrate this, the (two-dimensional) "mathematical construction plan" for our world, as I have defined it with my "intellectual game" in the beginning, provides us with the "Golden Section" $(G S)$, that is with the number sequence 618 i.e. the ratio 1.618 to 1 .

The actually measured value for the elementary charge is $1.60217733 \cdot 10^{-19}$ (C) $\beta$. The deviation amounts to a mere $0.99 \%$.

6) And finally we arrive at the so-called fine structure constant $\alpha$, which determines the limit for distances between each two smallest physical building blocks.

If its value were not $1: 137.0359895$ ( \pm a bit!) then atoms could not combine to molecules as they do. Instead of water, metal, stone and sand, for example, we would have nothing but a hotchpotch of atoms.

Thus, it also seems to have something to do with the number sequence for the limits of expansion, the feasibility $\Omega$, which is the number 273 . This again has something to do with the force between 2 bodies so that, analogous to the other constants, the factor 2:273 must logically be used.

After a simple reduction of this value we arrive at $1: 136.5$, which shows a mere $0.39 \%$ deviation from the actual measured value.

7) In the strict sense the numbers 273 and 618 are not natural constants; nonetheless they are the constants for perfection and the limit of feasibility and are often misjudged. In some of my books I acknowledged their importance and I was the first to demonstrate their derivation by very simple geometrical considerations. In several of my books I also formulated the apt metaphor for the creation of spirit and matter out of an indescribable divinity: via "i" at first "- 1 " is created and stands for the "spiritual world", then "+1", standing for the coming-up world of matter and finally $(+1)^{2}$, standing for a true spatial four-dimensionality of a real existing infinite space, by simple squaring (see references below).

Modern scientists ascribed a real existence only to physical perceptions. If we regard the world within these parameters a multitude of observations indicate that the number sequence 273 always marks the limits while 618 always proves to be the value for perfection and optimum.

Everything physical is subject to regular growth and disintegration which can be described by Euler's number $(e=2.72 \ldots)$. This means there are two limits of physical existence, one "upper" and one "lower" limit.

The number sequence 273 is derived from the geometrical ratio between the new square and the initial circle $(=1.273 \ldots)$, in my simple intellectual experiment concerning the development of the world. 1.618 ...

The Golden Section is thereby also created with

Transcribed to my metaphor mentioned above it follows: $(1.273 \ldots)^{2} \approx 1.618$ and $(1.618)^{2} \approx \mathrm{e}$.

\section{Conclusion}

Our world is most certainly an integrated whole. And as a result of my here shown demonstrations and some other aspects I dealt with in several books the current view of our world should indeed be changed drastically. The materialism, so characteristic of our times, cannot be the right notion.

\section{Notes}

1. Laack, W. van, “To Perceive The World With Logic”, ISBN 978-3-936624-08-3 (2007), see my little "history of creation", chapter 2.4. "From Singularity to Multiplicity"

2. $\mathrm{J}=$ Joule is also a decimal unit for energy. It is $1 \mathrm{~J}=1 \mathrm{Nm}$ (Newtonmeter) $=10 \mathrm{kgm} 2 / \mathrm{s} 2$

3. $\mathrm{C}=$ Coulomb $=\mathrm{As}=$ Ampère second, which is a decimal measure of the quantity of electricity.

\section{References}

Laack, W. van (2007). To Perceive the World with Logic, ISBN 978-3-936624-08-3 (SC), and as E-Book ISBN 978-3936624-09-0

Laack, W. van (2005). Nobody Ever Dies!, ISBN 3-936624-03-8 (SC), and as E-Book ISBN 978-3-936624-22-9

Laack, W. van (2001). A Better History of Our World, Vol. 1, The Universe, ISBN 3-8311-1490-0

Laack, W. van (2000). Key to Eternity, ISBN 3-8311-0344-5 